and dexamethasone on in vitro expression of lymphocyte function. Clin Exp Immunol 24: 54-62.

Grossman SA, Ye X, Lesser G, Sloan A, Carraway H, Desideri S, Piantadosi S (2011) Immunosuppression in patients with high-grade gliomas treated with radiation and temozolomide. Clin Cancer Res 17: 5473-5480.

Hughes MA, Parisi M, Grossman SA, Kleinberg L (2005) Primary brain tumors treated with steroids and radiotherapy: Low CD4 counts and risk of infection. Int J Radiat Oncol Biol Phys 62: 1423-1426.

Tang C, Liao Z, Gomez D, Levy L, Zhuang Y, Gebremichael RA, Hong DS, Komaki R, Welsh JW (2014) Lymphopenia associated with gross tumor volume and lung V5 and its effects on non-small cell lung cancer patient outcomes. Int J Radiat Oncol Biol Phys 89: 1084-1091.

Wild AT, Ye X, Ellsworth SG, Narang AK, Garg T, Campian J, Laheru DA, Zheng L, Wolfgang CL, Tran PT, Grossman SA, Herman JM (2015) The association between chemoradiation-related lymphopenia and clinical outcomes in patients with locally advanced pancreatic adenocarcinoma. Am J Clin Oncol 38: 259-265.

Wong ET, Lok E, Gautam S, Swanson KD (2015) Dexamethasone exerts profound immunologic interference on treatment efficacy for recurrent glioblastoma. Br J Cancer 113: 232-241.

${ }^{*}$ Correspondence: ET Wong; E-mail: ewong@bidmc.harvard.edu

Published online 8 September 2015

(c) 2015 Cancer Research UK. All rights reserved 0007-0920/15

\section{(c) (1) (2) (2)}

http://creativecommons.org/licenses/by-nc-sa/4.0/
BIC OPEN

\title{
BRAF-mutated metastatic colorectal cancer between past and future
}

\author{
Chiara Cremolini ${ }^{1}$, Massimo Di Maio ${ }^{2}$, Fausto Petrelli ${ }^{3}$, Rosa Berenato ${ }^{4}$, Fotios Loupakis ${ }^{1}$ and Filippo Pietrantonio*,4
}

${ }^{1}$ Polo Oncologico, Azienda Ospedaliero-Universitaria Pisana, Pisa, Italy; ${ }^{2}$ Dipartimento di Oncologia, Università degli studi di Torino, A.O.U. San Luigi Gonzaga, Orbassano, Italy; ${ }^{3}$ Azienda Ospedaliera Treviglio, Unità Operativa Oncologia, Treviglio, Bergamo, Italy and ${ }^{4}$ Medical Oncology Department, Fondazione IRCCS Istituto Nazionale dei Tumori, Milan, Italy

Sir,

We read with interest the meta-analysis by Rowland et al addressing the role of BRAF V600E mutation as predictor of benefit from anti-EGFR monoclonal antibodies (mAbs) in metastatic colorectal cancer.

Authors conclude that there is insufficient evidence to definitively state that RAS WT/BRAF MT individuals attain a reduced benefit from anti-EGFR mAbs compared with RAS WT/BRAF WT ones. Their conclusion is based on the lack of a significant interaction between $B R A F$ mutational status and the effect of the addition of an anti-EGFR mAb to standard therapies (Rowland et al, 2015)

In our opinion some considerations are needed to properly put these results in the clinical perspective, as pointed out in our previous work (Pietrantonio et al, 2015).

First, it should be noted that in terms of PFS, where the confounding effect of subsequent lines of treatment is absent, the $P$-value for interaction is equal to 0.07. Of note, an alfa-error up to 0.10 is often considered reasonable for interaction tests. In any case, it should be considered that these analyses are based on the retrospective, unplanned evaluation of subgroups of patients included in randomized trials and are therefore definitely underpowered to evidence a statistically significant difference. Although the global number of patients included in the analysis is high, the low incidence of BRAF V600E mutation weakens the power of this analysis. In the meta-analysis, OS comparison included 3096 patients (89\% BRAF wild-type and 11\% BRAF mutated). Even if $100 \%$ of events had been observed - that is a clear overestimation, especially with respect to OS data - the statistical power to detect a significant interaction between BRAF mutational status and the effect of anti-EGFR mAbs (assuming hazard ratio 0.8 in $B R A F$ wt and hazard ratio 1.0, that is, absence of effect, in $B R A F$ mutant patients) would have been as low as about $50 \%$. Therefore, even if the lack of statistical significance of the interaction test for OS is a matter of fact, the relevant risk of a false negative result should be properly acknowledged.

Second, results from FIRE-3 trial, comparing first-line FOLFIRI plus cetuximab with FOLFIRI plus bevacizumab were not included in the metanalysis by Rowland et al. In their discussion, authors elegantly argue that FIRE-3 is not sufficiently comparable to the other included trials, as bevacizumab use in the control arm is associated with a significant benefit, as compared with chemotherapy alone. We totally agree with that observation, but, again, by a practical perspective it should be recognised that first-line chemotherapy plus bevacizumab is one of the most common choices worldwide. From a clinical point of view, the decision of adding an antiEGFR mAb to chemotherapy in patients with $B R A F$ mutation, based on the absence of interaction between $B R A F$ status and treatment efficacy, would be totally reasonable in the absence of therapeutic alternatives. Given that an alternative is actually available, the use of an anti-EGFR mAb, instead of bevacizumab, should be probably reserved to those patients who may actually derive benefit from these drugs, with a different and often less acceptable toxicity profile. To this purpose, the metanalysis by the same authors highlighting the role of panRAS mutations as predictors of resistance to anti-EGFR mAbs, also including results from FIRE-3, is of special interest (Pietrantonio et al, 2015; Sorich et al, 2015). Unfortunately, results in the RAS WT/BRAF WT subgroup of the FIRE-3 trial have not been provided yet, thus preventing from including this trial in the present analysis. As information about $B R A F$ mutational status is also lacking from the other head-to-head randomized trials PEAK and CALGB80409, we recognise that the question about the 'best' biologic agent to be combined with a first-line chemotherapy doublet in BRAF mutant individuals is far from being answered.
Third, as BRAF mutant patients are often unable to receive subsequent lines of therapy (Seligmann et al, 2015), the choice of the upfront treatment is of paramount importance. Although results with doublets plus a biologic are disappointing (Stintzing et al, 2014), increasing evidences support the choice of FOLFOXIRI plus bevacizumab as a preferred option for fit patients (Fakih, 2015; Loupakis et al, 2014).

Nevertheless, more targeted approaches will hopefully enter the clinical scenario in the next future, based on promising results of early phase trials investigating BRAF \pm MEK and EGFR inhibitors in molecularly selected patients (Atreya et al, 2015). Knowing BRAF status is today crucial to allow $B R A F$ MT patients to enter clinical trials with those targeted agents.

In conclusion, although the negative predictive power of BRAF V600E mutation with respect to anti-EGFR mAbs will never be formally demonstrated in properly designed, wide and expensive clinical trials, $B R A F$ testing is today recommended by major guidelines. In our opinion, irrespectively of the personal choice of treating physicians to expose $B R A F$ mutant patients to antiEGFR mAbs, BRAF clearly stands as a molecular marker able to inform clinical decisions in the daily practice, and hopefully its role in treatment decisions will be better defined in the near future.

\section{CONFLICT OF INTEREST}

The authors declare no conflict of interest.

\section{REFERENCES}

Atreya CE, Van Cutsem E, Bendell JC, Andre T, Schellens JHM, Gordon MS,

McRee AJ, O’Dwyer PJ, Muro K, Tabernero J, van Geel R, Sidhu R, Greger JG Rangwala FA, Motwani M, Wu Y, Orford KW, Corcoran RB (2015) Updated efficacy of the MEK inhibitor trametinib (T), BRAF inhibitor dabrafenib (D), and anti-EGFR antibody panitumumab (P) in patients (pts) with BRAF V600E mutated (BRAFm) metastatic colorectal cancer (mCRC). J Clin Oncol 33(suppl 3): abstr 103 .

Fakih MG (2015) Metastatic colorectal cancer: current state and future directions. J Clin Oncol 33: 1809-1824.

Loupakis F, Cremolini C, Masi G, Lonardi S, Zagonel V, Salvatore L, Cortesi E Tomasello G, Ronzoni M, Spadi R, Zaniboni A, Tonini G, Buonadonna A, Amoroso D, Chiara S, Carlomagno C, Boni C, Allegrini G, Boni L, Falcone A (2014) Initial therapy with FOLFOXIRI and bevacizumab for metastatic colorectal cancer. $N$ Engl J Med 371: 1609-1618.

Pietrantonio F, Petrelli F, Coinu A, Di Bartolomeo M, Borgonovo K, Maggi C, Cabiddu M, Iacovelli R, Bossi I, Lonati V, Ghilardi M, de Braud F, Barni S (2015) Predictive role of BRAF mutations in patients with advanced colorectal cancer receiving cetuximab and panitumumab: a meta-analysis. Eur I Cancer 51: 587-594

Pietrantonio F, Cremolini C, Petrelli F, Di Bartolomeo M, Loupakis F, Maggi C, Antoniotti C, de Braud F, Falcone A, Iacovelli R (2015) First-line anti-EGFR monoclonal antibodies in panRAS wild-type metastatic colorectal cancer: A systematic review and meta-analysis. Crit Rev Oncol Hematol doi:10.1016/ j.critrevonc.2015.05.016.

Rowland A, Dias MM, Wiese MD, Kichenadasse G, McKinnon RA, Karapetis CS, Sorich MJ (2015) Meta-analysis of BRAF mutation as a predictive biomarker of benefit from anti-EGFR monoclonal antibody therapy for RAS wild-type metastatic colorectal cancer. Br J Cancer 112: 1888-1894.

Seligmann JF, Fisher D, Elliott F, Richman S, Butler R, Cheadle J, Adams RA, Quirke P, Maughan T, Seymour MT, Middleton GW (2015) Exploring the poor outcomes of BRAF mutant (BRAF mut) advanced colorectal cancer (aCRC): Analysis from 2,530 patients (pts) in randomized clinical trials (RCTs). I Clin Oncol 33(suppl): abstr 3509.

Sorich MJ, Wiese MD, Rowland A, Kichenadasse G, McKinnon RA, Karapetis CS (2015) Extended RAS mutations and anti-EGFR monoclonal antibody survival 
benefit in metastatic colorectal cancer: a meta-analysis of randomized, controlled trials. Ann Oncol 26: 13-21.

Stintzing S, Jung A, Rossius L, Modest DP, von Weikersthal LF, Decker T, Kiani A, Al-Batran SE, Vehling-Kaiser U, Heintges T, Moehler M, Scheithauer W, Kirchner T, Heinemann V (2014) Mutations within the
EGFR signaling pathway: Influence on efficacy in FIRE-3-A randomized phase III study of FOLFIRI plus cetuximab or bevacizumab as first-line treatment for wild-type (WT) KRAS (exon 2) metastatic colorectal cancer (mCRC) patients. J Clin Oncol 32(suppl 3): abstr 445 .

*Correspondence: F Pietrantonio; E-mail: filippo.pietrantonio@istitutotumori.mi.it

Published online 10 September 2015

(c) 2015 Cancer Research UK. All rights reserved 0007 - 0920/15
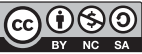

http://creativecommons.org/licenses/by-nc-sa/4.0/

\title{
Reply: Comment on 'Meta-analysis of BRAF mutation as a predictive biomarker of benefit from anti-EGFR monoclonal-antibody therapy for RAS wild-type metastatic colorectal cancer'
}

\author{
Andrew Rowland ${ }^{\star}, 1,2$, Mafalda M Dias ${ }^{1,3}$, Michael D Wiese ${ }^{3}$, Ganessan Kichenadasse ${ }^{2}$, Ross A McKinnon², Christos S Karapetis ${ }^{2}$ \\ and Michael J Sorich ${ }^{1,2}$
}

\begin{abstract}
${ }^{1}$ Department of Clinical Pharmacology, School of Medicine, Flinders University, Adelaide 5042, South Australia, Australia; ${ }^{2}$ Flinders Centre for Innovation in Cancer, School of Medicine, Flinders University, Adelaide 5042, South Australia, Australia and ${ }^{3}$ School of Pharmacy and Medical Sciences, University of South Australia, Adelaide 5000, South Australia, Australia
\end{abstract}

Sir,

We thank Pietrantonio et al, for their interest in our recent manuscript (Rowland et al, 2015). We also acknowledge their mutual interest in the field, and share their goal of informing clinicians with the most accurate and complete evidence to ensure that patients with metastatic colorectal cancer (mCRC) are both afforded access and guided to the most appropriate treatment interventions.

Pietrantonio et al, highlight that the power to detect differences in treatment effect between subgroups based on BRAF mutation status is often poor, and imply that the risk of false negative results is not properly acknowledged in this manuscript. As described in the discussion section of the manuscript, we have clearly and prominently addressed the issue of false positive and negative results (Rowland et al, 2015). We believe that the evaluation of the predictive nature of BRAF mutation for anti-EGFR therapy highlights what may be a relatively common scenario in which it will be difficult to make definitive conclusions despite having results from a number of high quality secondary analyses of clinical trials. Factors contributing to the substantial risk of false positive or false negative conclusions in this setting include (i) the post hoc nature of the analyses and the associated difficulty in correcting for multiple hypotheses testing, (ii) the biomarker having a low prevalence impacting on the precision of the estimates, (iii) there being significant statistical heterogeneity (inconsistency) in results between clinical studies, and (iv) that this biomarker may have a more modest impact (e.g. attenuate than annul) on treatment efficacy compared to the prominent biomarkers that have made their way into routine clinical practice such as RAS mutations (Sorich et al, 2014).

Pietrantonio et al. highlight their own meta-analysis of anti-EGFR mAb therapy in BRAF mutant tumours (Pietrantonio et al, 2015). This meta-analysis concludes that ' $\mathrm{C}$ - or P-based therapy did not increase the benefit of standard therapy or the BSC in RAS-wt/BRAF-mut CRC patients.' This meta-analysis principally differs from ours on the basis of the methodology used to evaluate whether BRAF is a predictive marker of anti-EGFR mAb efficacy. The evaluation of heterogeneity of effect between subgroups by a test of interaction is the standard approach recommended, on the basis that evaluating the efficacy of a treatment with respect to an isolated subgroup is well known to have a high risk of false positive results (i.e. falsely concluding that a subgroup has no effect) (Rothwell, 2005; Kent et al, 2010; Sun et al, 2010). The Pietrantonio meta-analysis only evaluated anti-EGFR efficacy in the BRAF mutant subgroup, whereas our study compared the efficacy in the BRAF mutant subgroup to the subgroup without a BRAF mutation (see (Altman and Matthews, 1996; Matthews and Altman, 1996) for a simple introduction to the concept of interaction). With respect to significance level, in our experience a stricter rather than more lenient significance level is often preferred for making strong claims that will have significant clinical and policy implications (as compared with exploratory/ screening questions). This is due to the post hoc nature of many subgroup analyses and the inflated risks of false positives with multiple hypotheses testing which are generally not explicitly adjusted for (Rothwell et al, 2005).

Our analysis highlight that the evidence for there being a treatment effect difference between BRAF subgroups does not meet the conventional levels of evidence when evaluated using the generally accepted approach for evaluating subgroup differences in RCTs-hence our more moderate conclusion that there currently is insufficient evidence to definitively state that there is a reduced (or no) benefit for individuals with mutated BRAF. Cognizant of the risk of false negative results, we have not ruled out the possibility that BRAF mutation status influences anti-EGFR therapy efficacy, merely that the evidence does not support a definitive claim that BRAF mutations does impact on efficacy. We advocate that as we can neither definitively claim or rule out a predictive effect of a BRAF mutation that it should remain at the clinician's and patient's discretion to decide whether to test for BRAF mutation and whether use of an anti-EGFR $\mathrm{mAb}$ is appropriate for a specific patient with a BRAF mutant tumor. We are concerned that the conclusion of the Pietrantonio meta-analysis of no benefit for the BRAF mutant subgroup may inadvertently lead to reduced clinician discretion to treat patients with BRAF mutant tumours. For example, if the evidence clearly indicated that anti-EGFR mAbs do not have benefit for patients with BRAF mutant tumours, then in many jurisdictions this would lead payers to restrict subsidy of anti-EGFR mAbs to individuals without a BRAF mutation (with routine testing for BRAF mutations). We do not believe that the evidence currently available supports with sufficient certainty that such individuals do not receive any benefit from anti-EGFR mAb therapy.

We agree with Pietrantonio et al, that the trials comparing bevacizumab to anti-EGFR $\mathrm{mAb}$ therapy are very informative in terms of guiding contemporary first line therapy in mCRC and that when results stratified by BRAF mutations status become available they will provide additional useful insight into the impact of BRAF mutations on anti-EGFR mAb therapy. However, it will be important to carefully manage how this data is analysed in conjunction with the data from trials that evaluate the addition of anti-EGFR therapy to standard therapy (e.g. focus on the difference between subgroups (Sorich et al, 2014)) as pooling results from these different types of trials in a meta-analysis focusing only on the effect size in the BRAF mutant subgroup may be misleading (Rowland et al, 2015).

On behalf of my colleagues

Kind Regards

Dr A Rowland

\section{CONFLICT OF INTEREST}

The authors declare no conflict of interest.

\section{REFERENCES}

Altman DG, Matthews JNS (1996) Statistics Notes: interaction 1: heterogeneity of effects. BMJ 313: 808.

Kent DM, Rothwell PM, Ioannidis JP, Altman DG, Hayward RA (2010) Assessing and reporting heterogeneity in treatment effects in clinical trials: a proposal. Trials 11: 85 .

Matthews JNS, Altman DG (1996) Statistics Notes: Interaction 2: compare effect sizes not $\mathrm{P}$ values. $B M J$ J13: 313 .

Pietrantonio F, Petrelli F, Coinu A, Di Bartolomeo M, Borgonovo K, Maggi C Cabiddu M, Iacovelli R, Bossi I, Lonati V, Ghilardi M, de Braud F, Barni S (2015) Predictive role of BRAF mutations in patients with advanced colorectal cancer receiving cetuximab and panitumumab: a meta-analysis. Eur J Cancer 51: $587-594$.

Rothwell PM (2005) Treating individuals 2. Subgroup analysis in randomised controlled trials: importance, indications, and interpretation. Lancet 365: $176-186$.

Rowland A, Dias MM, Wiese MD, Kichenadasse G, McKinnon RA, Karapetis CS, Sorich MJ (2015) Meta-analysis of BRAF mutation as a predictive biomarker of benefit from anti-EGFR monoclonal antibody therapy for RAS wild-type metastatic colorectal cancer. Br J Cancer 112: 1888-1894.

Sorich MJ, Wiese MD, Rowland A, Kichenadasse G, McKinnon RA, Karapetis CS (2014) Extended RAS mutations and anti-EGFR monoclonal antibody survival benefit in metastatic colorectal cancer: a meta-analysis of randomized controlled trials. Ann Oncol 26: 13-21.

Sun X, Briel M, Walter SD, Guyatt GH (2010) Is a subgroup effect believable? Updating criteria to evaluate the credibility of subgroup analyses. $B M J$ 340: c117

${ }^{\star}$ Correspondence: Dr A Rowland; E-mail: andrew.rowland@flinders.edu.au Published online 10 September 2015

(c) 2015 Cancer Research UK. All rights reserved 0007-0920/15

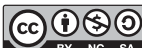

